\begin{tabular}{|c|l|}
\hline Title & $\begin{array}{l}\text { A New Brain Positron Emission Tomography Scanner with Semiconductor Detectors for Target Volume Delineation } \\
\text { and Radiotherapy Treatment Planning in Patients with Nasopharyngeal Carcinoma. }\end{array}$ \\
\hline Author(s) & $\begin{array}{l}\text { Katoh, Norio; Y asuda, Koichi; Shiga, Tohru; Hasegawa, Masakazu; Onimaru, Rikiya; Shimizu, Shinichi; Bengua, } \\
\text { Gerard; Ishikawa, Masay ori; Tamaki, Nagara; Shirato, Hiroki }\end{array}$ \\
\hline Citation & $\begin{array}{l}\text { International journal of radiation oncology, biology, phy sics, 82(4), e671-e676 } \\
\text { https://doi.org/40.1016/.jirobp.2011.09.011 }\end{array}$ \\
\hline Issue Date & 2012-03-15 \\
\hline Doc URL & http:/hdl.handle.net/2115/48568 \\
\hline Type & article(author version) \\
\hline File Information & IJROBP82-4_e671-e676_katoh_rev.pdf \\
\hline
\end{tabular}

Instructions for use 


\section{A New Brain Positron Emission Tomography (PET) Scanner with Semiconductor \\ Detectors For Target Volume Delineation and Radiotherapy Treatment Planning \\ In Patients with Nasopharyngeal Carcinoma}

Norio Katoh, M.D., Koichi Yasuda, M.D., ${ }^{*}$ Tohru Shiga, M.D., ${ }^{\dagger}$ Masakazu Hasegawa, M.D., Rikiya Onimaru, M.D., ${ }^{*}$ Shinichi Shimizu, M.D., ${ }^{*}$ Gerard Bengua, Ph.D., ${ }^{\ddagger}$ Masayori Ishikawa, Ph.D., ${ }^{\star}$ NagaraTamaki, M.D., ${ }^{\dagger}$ Hiroki Shirato, M.D. ${ }^{*}$

Department of Radiology*, Department of Nuclear Medicine**, Department of Medical Physics***, Hokkaido University Graduate School Medicine, Sapporo, Japan

\section{Address for reprints:}

Norio Katoh, M.D., Department of Radiology, Hokkaido University Graduate School of Medicine, North-15 West-7, Kita-ku, Sapporo, Japan, 060-8638

Tel: 81-11-706-5977, Fax: 81-11-706-7876, E-mail: noriwokatoh@med.hokudai.ac.jp

Running title: A new brain PET for radiotherapy planning in patients with NPC

Acknowledgements: This study was supported in part by the Ministry of Health, Labour, and Welfare and by the Ministry of Education, Culture, Sports, Science, and Technology, Japan. 
Katoh 2

\section{CONFLICTS OF INTEREST NOTIFICATION}

Any actual or potential conflicts of interest do not exist. 


\section{SUMMARY}

Two treatment planning methods for nasopharyngeal carcinoma (NPC) were compared: conventional whole-body BGO scintillator positron emission tomography (PET) $\left(\mathrm{PET}_{\mathrm{CONV}} \mathrm{WB}\right)$ and a new brain PET system using semiconductor detectors (PET $\mathrm{NEWBR}_{\mathrm{B}}$. In this study, 12 patients with NPC were analyzed. The gross tumor volume (GTV) was visually delineated on PET images using either PET $_{\mathrm{CONV}} \mathrm{WB}$ or

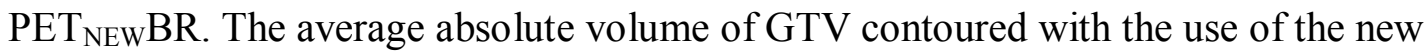
brain PET was significantly smaller than that of conventional whole-body BGO PET. Assuming a stereotactic radiotherapy boost plan of 7 ports, the plan using the new brain PET would significantly reduce the maximum dose to the cerebrum and cerebellum and brain stem. The new brain PET system using semiconductor detectors can provide more accurate tumor delineation than the conventional whole-body BGO PET system and has the potential to offer functional and molecular radiotherapy treatment planning. 


\begin{abstract}
Purpose: We compared treatment planning of stereotactic boost for nasopharyngeal carcinoma (NPC) between using conventional whole-body BGO scintillator positron emission tomography (PET) (PET $\left.{ }_{\mathrm{CONV}} \mathrm{WB}\right)$ and using the new brain PET system using semiconductor detectors $\left(\mathrm{PET}_{\mathrm{NEW}} \mathrm{BR}\right)$.

Methods and Materials: The present study included 12 patients with NPC. ${ }^{18}$ F-fluorodeoxyglucose-PET images were acquired with both the $\mathrm{PET}_{\mathrm{NEW}} \mathrm{BR}$ and a

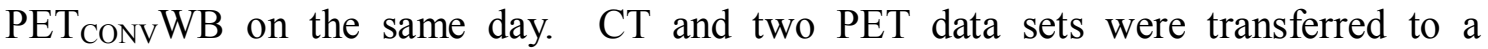
treatment planning system, and the $\mathrm{PET}_{\mathrm{CONV}} \mathrm{WB}$ and $\mathrm{PET}_{\mathrm{NEW}} \mathrm{BR}$ images were co-registered with the same set of CT images. The window width and level for all PET images were fixed at 3000 and 300 respectively. The gross tumor volume (GTV) was visually delineated on PET images using either PET $_{\text {CONV }} \mathrm{WB}$ images $\left(\mathrm{GTV}_{\mathrm{CONV}}\right)$ or PET $_{\text {NEW }} B R$ images $\left(\mathrm{GTV}_{\mathrm{NEW}}\right)$. Assuming a stereotactic radiotherapy boost of 7 ports, the prescribed dose delivered to $95 \%$ of the planning target volume (PTV) was set to 2000 cGy in 4 fractions.
\end{abstract}

Results: The average absolute volume of $\mathrm{GTV}_{\mathrm{NEW}}$ was $15.7 \mathrm{ml}$ (standard deviation; SD, 9.9), and that of $\mathrm{GTV}_{\mathrm{CONV}}$ was $34.0 \mathrm{ml}(\mathrm{SD}, 20.5)$. The average of $\mathrm{GTV}_{\mathrm{NEW}}$ was significantly smaller than that of $\operatorname{GTV}_{\mathrm{CONV}}(\mathrm{p}=0.0006)$. There was no statistically 
significant difference in the maximum dose $(p=0.0585)$ and mean dose $(p=0.2748)$ of

PTV. PLAN $\mathrm{NEW}_{\mathrm{N}}$ significantly reduced the maximum dose of cerebrum and cerebellum $(\mathrm{p}=0.0418)$ and of brain stem $(\mathrm{p}=0.0041)$.

Conclusion: The present study suggests that the new brain PET system using semiconductor detectors can provide more accurate tumor delineation than conventional whole-body BGO PET system and may be an important tool for functional and molecular radiotherapy treatment planning.

Key words: semiconductor, positron emission tomography, radiotherapy planning, target volume delineation, nasopharyngeal carcinoma 


\section{INTRODUCTION}

Since the advent of computed tomography (CT), sophisticated techniques in radiation treatment such as three-dimensional conformal radiotherapy, stereotactic radiotherapy, and intensity-modulated radiotherapy (IMRT) have been developed in order to focus and escalate the radiation dose to the tumor while sparing normal tissues. In these techniques, it is important to precisely determine the tumor volume. With their high anatomic resolutions, $\mathrm{CT}$ and magnetic resonance imaging (MRI) have been primarily used for target volume delineation in radiotherapy treatment planning. However, when delineating the target volume, it is sometimes difficult to distinguish between tumor and non-tumor tissues using anatomical imaging alone. In the past 10 years, positron emission tomography (PET) with ${ }^{18}$ F-fluorodeoxyglucose (FDG), which is able to visualize molecular information for the tumor, has been widely used in oncology for the diagnosis and staging of various cancers. This functional imaging has been adopted in radiotherapy, and several studies have examined the clinical impact of PET on radiotherapy planning (1-3). However, since PET is not an intrinsically accurate examination, with a spatial resolution of approximately 4 to $7 \mathrm{~mm}(4-6)$, it is difficult to determine tumor boundaries on conventional seintillater whole-body BGO scintillator PET images. In 2007, a new brain PET scanner with semiconductor detectors, the first 
in the world, was developed with HITACHI, Ltd and was installed at our institute (7). This brain PET system equipped with small semiconductor detectors and depth of interaction (DOI) system to obtain sufficient sensitivity and a higher spatial resolution (2.3 mm at $1 \mathrm{~cm}$ (NEMA NU 2-2001)). Semiconductor detectors also have an advantage in energy resolution. Our new semiconductor PET detectors had an energy resolution of $4.1 \%$ (FWHM) which is superior to the energy resolution obtained with available scintillation detectors (e.g., 10\%-20\%) $(8,9)$. The limited energy window set permits the collection of accurate signal counts with lower noise counts. The scatter fraction of the new brain PET system was 23\% (NEMA NU 2-1994), which was lower than those of other, scintillation-based whole-body BGO PET scanners such as EXACT HR+ (Asahi-Siemens, Tokyo, Japan) (32.1\% (NEMA NU 2-1994)) $(10,11) . \quad$ In our previous study, the contrasts obtained with the semiconductor brain PET scanner was $27 \%$ higher than that obtained with the conventional whole-body BGO scanner for both a cold spot phantom that had 6-mm-diameter cold sphenoid defects, a dual-cylinder phantom with an adjusted concentration of 1:2 surrounded with water (7). For patients with nasopharyngeal carcinoma (NPC), the new brain PET system identified intratumoral inhomogeneity in more detail than the conventional whole-body BGO PET system and the tumor edge was sharper on the images obtained with the new brain PET system than 
on those obtained with the conventional whole-body BGO PET system (7). Therefore, the new brain PET system has the potential to provide high contrast and detailed images with sharper tumor edge in radiation treatment planning for NPC.

The purpose of this study was to evaluate the effect of the use of the new brain PET system for radiotherapy treatment planning of patients with NPC comparing with a conventional whole-body BGO PET, EXACT HR+.

\section{METHODS AND MATERIALS}

\section{Patients}

The subjects considered in this study were 12 NPC patients who had been newly diagnosed from July 2007 to April 2009. The median age was 61 years old (range 30-76 years old). The patient characteristics are shown in Table 1. Written informed consent was obtained from all patients.

\section{Image acquisition / Target volume delineation}

Before the PET study, all patients fasted for at least $6 \mathrm{~h}$. Serum glucose levels were checked in all of the patients before the administration of ${ }^{18}$ F-FDG. The dose of ${ }^{18} \mathrm{~F}$-FDG for each patient was $370 \mathrm{MBq} .{ }^{18}$ F-FDG-PET images were acquired in a diagnostic, nontreatment position with the new brain PET system using semiconductor detectors (PET $\mathrm{NEW}_{\mathrm{NR}}$ ) and with a conventional whole-body BGO PET system 


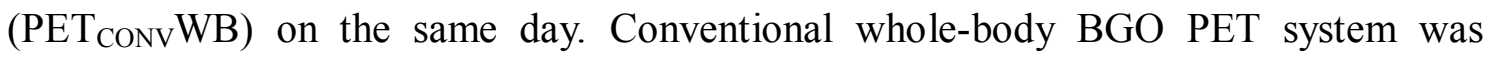
performed using EXACT $\mathrm{HR}+$. Two time-course protocols were adopted and randomly selected. In Protocol 1, $\mathrm{PET}_{\mathrm{CONV}} \mathrm{WB}$ images were acquired first, and in Protocol 2, $\mathrm{PET}_{\mathrm{NEW}} \mathrm{BR}$, images were acquired first. Among the 12 patients, there were 7 in Protocol 1 and 5 in Protocol 2. The difference in the distribution was that the time-course protocols were used for all patients who received the $\mathrm{PET}_{\mathrm{NEW}} \mathrm{BR}$ scans, not just patients with NPC but also those with brain tumors, epilepsy, etc.

CT with a slice thickness of $2-5 \mathrm{~mm}$ was performed. The CT and two PET data sets were transferred to the Pinnacle ${ }^{3}$ treatment planning system (version 8.0; Philips Medical Systems, Fitchburg, WI) for image registration, target volume delineation, and volume analysis. The $\mathrm{PET}_{\mathrm{CONV}} \mathrm{WB}$ and $\mathrm{PET}_{\mathrm{NEW}} \mathrm{BR}$ images were co-registered with the same set of $\mathrm{CT}$ images. $\mathrm{PET}_{\mathrm{NEW}} \mathrm{BR}$ images on the Pinnacle ${ }^{3}$ treatment planning system were not displayed using the standardized uptake value scales for window level/width; as such, we used the raw value scales, and the window width and level in all PET images were fixed at 3000 and 300, respectively. The gross tumor volume (GTV) was visually delineated on PET images alone by an experienced nuclear medicine physician and a radiation oncologist in consensus. When drawing the GTV contour, CT images were not used. Since the new brain PET scanner with semiconductor detectors is 
dedicated to brain imaging, the bottom level of PET $_{\mathrm{CONV}} \mathrm{WB}$ images used in this study were adjusted to almost the same that of PET $_{\text {NEW }} B R$ images; the GTV was limited to the primary tumors and/or retropharyngeal lymph nodes in this study. GTV $\mathrm{CONV}_{\text {was }}$ determined using PET $_{\mathrm{CONV}} \mathrm{WB}$ images, while $\mathrm{GTV}_{\mathrm{NEW}}$ was determined using PET $_{\mathrm{NEW}} \mathrm{BR}$ images. There was an interval of approximately a week between the

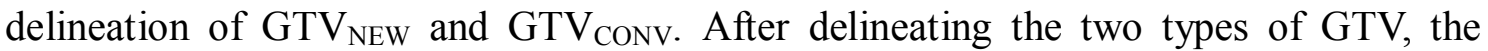
cerebrum and cerebellum and the brain stem were contoured on CT images.

\section{Radiotherapy treatment planning simulation}

The clinical target volume (CTV) was defined three-dimensionally as the GTV with a 2-mm margin, while the planning target volume (PTV) was defined as the CTV plus a 3-mm margin. Assuming a stereotactic radiotherapy boost of 7 ports, the prescribed dose delivered to $95 \%$ of PTV was set to 2000 cGy in 4 fractions. A radiotherapy treatment plan was prepared for $\mathrm{GTV}_{\mathrm{NEW}}$ and $\mathrm{GTV}_{\mathrm{CONV}}$. Dose-volume histograms (DVHs) were calculated for the PTV, the cerebrum and cerebellum, and the brain stem in both plans.

\section{Statistical analysis}

Absolute volumes of GTV and DVH parameters were compared. The difference was evaluated using the paired t-test. $P<0.05$ was considered statistically significant. 


\section{RESULTS}

Absolute volumes of $\mathrm{GTV}_{\mathrm{NEW}}$ and $\mathrm{GTV}_{\mathrm{CONV}}$ are shown in Table 2. The average absolute volume of $\mathrm{GTV}_{\mathrm{NEW}}$ was $15.7 \mathrm{ml}$ (standard deviation; SD, 9.9, range, 4.9 -31.6), and that of $\mathrm{GTV}_{\mathrm{CONV}}$ was $34.0 \mathrm{ml}(\mathrm{SD}, 20.5$, range, 10.6 - 75.9). The average absolute volume of $\mathrm{GTV}_{\mathrm{NEW}}$ was significantly smaller than that of $\mathrm{GTV}_{\mathrm{CONV}}(\mathrm{p}=0.0006)$. Regardless of the order of two ${ }^{18}$ F-FDG examinations, volumes of $\mathrm{GTV}_{\mathrm{NEW}}$ were always smaller than $\mathrm{GTV}_{\mathrm{CONV}}$ for all 12 patients.

The maximum and mean doses of $\mathrm{PTV}_{\mathrm{NEW}}$ and $\mathrm{PTV}_{\mathrm{CONV}}$ are shown in Table 3 . There was no statistically significant difference in the maximum dose $(p=0.0585)$ or the mean dose $(p=0.2748)$. The maximum doses for cerebrum and cerebellum $(\mathrm{CC})$ and

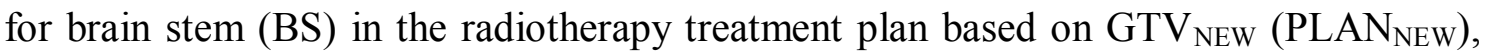
and those in the plan based on $\operatorname{GTV}_{\mathrm{CONV}}\left(\mathrm{PLAN}_{\mathrm{CONV}}\right.$ ) are shown in Table 4 . In PLAN $_{\mathrm{NEW}}$, the average maximum dose of CC was 2001 cGy (SD, 347, range, 1278 -2430) and that of BS was 1475 cGy (SD, 612, range, 586 - 2243. In PLAN CONV $_{\text {, the }}$ average maximum dose of CC was $2233 \mathrm{cGy}(\mathrm{SD}, 209$, range, $1627-2442)$ and that of BS was 1816 cGy (SD, 455, range, $664-2197)$.

Compared with PLAN ${ }_{\mathrm{CONV}}$, PLAN $\mathrm{NEW}_{\mathrm{N}}$ significantly reduced the maximum dose of CC 
$(p=0.0418)$ and BS $(p=0.0041)$. An example of PLAN NEW $_{\text {and PLAN }}$ anV is shown in

Figs. 1 and 2.

\section{DISCUSSION}

Although PET offers better identification of tumor localization than the anatomical imaging modalities because of its higher contrast resolution, tumor boundaries are blurred on conventional BGO PET system because of its relatively low spatial resolution due to its larger detectors and worse annihilation non-collinearity blurring because of the larger detector ring of whole-body BGO PET. Daisne et al. have reported that the PET-derived volumes are more accurate than CT or MRI-derived volumes for squamous cell carcinoma of the head and neck; however, they are still larger than those delineated from the surgical specimens (12).

We did not use CT images when delineating the GTV in order to evaluate the impact of the difference of the two PET scanners on radiotherapy treatment planning. The present study has shown that the absolute GTV volumes on PET $_{\mathrm{NEW}} \mathrm{BR}$ system are significantly smaller than those on $\mathrm{PET}_{\mathrm{CONV}} \mathrm{WB}$ system, and that the smaller size of the GTV on $\mathrm{PET}_{\mathrm{NEW}} \mathrm{BR}$ is not likely due to the time of examination. There are several potential reasons why the GTV is smaller for the new brain PET system using semiconductor 
detectors. One main reason is the difference of the spatial resolution of the two PET systems. Higher spatial resolution yielded shaper edge of the tumor without doubt (7). Additional possible reasons were lower scatter fraction and higher contrast of the $\mathrm{PET}_{\mathrm{NEW}} \mathrm{BR}$ system $(8-11)$. Further study is needed to determine how much geometry of the detectors, energy resolution of the semiconductor detector, reconstruction algorism, and other mechanical factors were influential quantitatively on the size of GTV.

In the simulation of radiotherapy treatment planning, this target volume reduction resulted in a decrease in the radiation dose to organs at risk such as $\mathrm{CC}$ and $\mathrm{BS}$. Although we did not compare the pathologic specimens to the target volumes on PET images and it is unclear whether the PET $_{\mathrm{NEW}} \mathrm{BR}$-based GTV accurately reflected the true tumor volume, we consider the reduction of absolute GTV volumes to be primarily due to the tumor edge on the $\mathrm{PET}_{\mathrm{NEW}} \mathrm{BR}$ image being more clearly defined. However, this reduction of GTV volumes might be smaller if CT images were used with both PET images for the delineation of GTV.

We adopted a visual interpretation method for the delineation of GTV. This method is commonly used (13-17) but is influenced by the display windowing and is dependent on operators. Therefore, several objective methods for contouring PET images have been 
developed, including isocontouring based on a fixed threshold of a standardized uptake value $(1,17-20)$, a fixed threshold of $40 \%$ to $50 \%$ of the maximum activity $(3,17$, 20-22), and a threshold adapted to the signal-to-background ratios $(2,12,17)$. However, the appropriate standardized technique for the segmentation of PET images is still under investigation in the head and neck region $(4-6,23-26)$. It is probable that the lack of a standardized method for segmentation is due in part to the intrinsically low quality of PET images. As such, PET $_{\mathrm{NEW}} \mathrm{BR}$ images could lead to a new standardized segmentation method, and we consider it necessary to evaluate the interobserver variability of the target delineation and to compare objective segmentation methods for the $\mathrm{PET}_{\mathrm{NEW}} \mathrm{BR}$ images.

Another limitation is that we did not compare our new brain PET results with state of the art brain PET system such as Siemens HRRT, but just compared with relatively old whole-body camera, Siemens HR + system with a standard OSEM reconstruction method. We would like to stress the advantages of new brain PET camera with higher resolution and less scatter noise which may facilitate delineation of tumor for radiation therapy than the conventional whole-body BGO PET camera. However, HR+ system provides relatively high-resolution PET images with the current reconstruction algorithm. We are now planning to develop a next prototype PET camera with wide 
aperture and high sensitivity. We consider it necessary to compare a state-of-the-art LSO PET scanner with our new PET in the future.

We have previously reported that the $\mathrm{PET}_{\mathrm{NEW}} \mathrm{BR}$ scanner has the potential to provide better identification of intratumoral inhomogeneity (7). It is likely that IMRT can accurately deliver a higher dose to the lesion with higher intratumoral uptake on the new brain PET system using semiconductor detectors. In addition to ${ }^{18} \mathrm{~F}-\mathrm{FDG}$, there are various tracers related to tumor cell hypoxia, proliferation, or metabolism $(4,26)$. If the PET $_{\text {NEW }} \mathrm{BR}$ images with these tracers are incorporated into IMRT planning, functional and molecular target radiotherapy will become practicable.

\section{CONCLUSION}

Our results suggest that compared to conventional whole-body BGO PET system, the new brain PET system using semiconductor detectors can provide better identification of tumor boundaries and more accurate tumor delineation; as such, it may be an important tool for functional and molecular radiotherapy treatment planning. 


\section{REFERENCES}

1. Vernon MR, Maheshwari M, Schultz CJ, et al. Clinical outcomes of patients receiving integrated PET/CT-guided radiotherapy for head and neck carcinoma. Int J Radiat Oncol Biol Phys 2008;70:678-684.

2. Madani I, Duthoy W, Derie C, et al. Positron emission tomography-guided, focal-dose escalation using intensity-modulated radiotherapy for head and neck cancer. Int J Radiat Oncol Biol Phys 2007;68:126-135.

3. Guido A, Fuccio L, Rombi B, et al. Combined 18F-FDG-PET/CT imaging in radiotherapy target delineation for head-and-neck cancer. Int J Radiat Oncol Biol Phys 2009;73:759-763.

4. Ford EC, Herman J, Yorke E, et al. 18F-FDG PET/CT for image-guided and intensity-modulated radiotherapy. $J$ Nucl Med 2009;50:1655-1665.

5. MacManus M, Nestle U, Rosenzweig KE, et al. Use of PET and PET/CT for radiation therapy planning: IAEA expert report 2006-2007. Radiother Oncol 2009;91:85-94.

6. Ahn PH, Garg MK. Positron emission tomography/computed tomography for target delineation in head and neck cancers. Semin Nucl Med 2008;38:141-148. 
7. Shiga T, Morimoto Y, Kubo N, et al. A new PET scanner with semiconductor detectors enables better identification of intratumoral inhomogeneity. $J$ Nucl Med 2009;50:148-155.

8. Yanagishita N, Morimoto Y, Ishitsu T, et al. Physical performance of a prototype 3D PET scanner using CdTe detectors. Nuclear Science Symposium Conference Record, 2007. Vol 4. Piscataway (NJ): IEEE; 2007; 2665-2668.

9. Morimoto Y, Ueno Y, Kobashi K, et al. Performance of a prototype brain PET scanner based on semiconductor detectors [Abstract]. J Nucl Med 2008;49 (Suppl. $1): 122 \mathrm{P}$

10. Daube-Witherspoon ME, Karp JS, Casey ME, et al. PET performance measurements using the NEMA NU 2-2001 standard. $J$ Nucl Med 2002;43:1398-1409

11. Adam L-E, Zaers J, Ostertag H, et al. Performance evaluation of the whole-body PET scanner ECAT EXACT HR+ following the IEC standard. IEEE Trans Nucl Sci 1997;44:1172-1179

12. Daisne JF, Duprez T, Weynand B, et al. Tumor volume in pharyngolaryngeal squamous cell carcinoma: comparison at CT, MR imaging, and FDG PET and validation with surgical specimen. Radiology 2004;233:93-100. 
13. Heron DE, Andrade RS, Flickinger J, et al. Hybrid PET-CT simulation for radiation treatment planning in head-and-neck cancers: a brief technical report. Int J Radiat Oncol Biol Phys 2004;60:1419-1424.

14. Riegel AC, Berson AM, Destian S, et al. Variability of gross tumor volume delineation in head-and-neck cancer using CT and PET/CT fusion. Int J Radiat Oncol Biol Phys 2006;65:726-732.

15. Nishioka T, Shiga T, Shirato H, et al. Image fusion between 18FDG-PET and $\mathrm{MRI} / \mathrm{CT}$ for radiotherapy planning of oropharyngeal and nasopharyngeal carcinomas. Int J Radiat Oncol Biol Phys 2002;53:1051-1057.

16. Ciernik IF, Dizendorf E, Baumert BG, et al. Radiation treatment planning with an integrated positron emission and computer tomography (PET/CT): a feasibility study. Int J Radiat Oncol Biol Phys 2003;57:853-863.

17. Schinagl DA, Vogel WV, Hoffmann AL, et al. Comparison of five segmentation tools for 18F-fluoro-deoxy-glucose-positron emission tomography-based target volume definition in head and neck cancer. Int J Radiat Oncol Biol Phys 2007;69:1282-1289.

18. Nestle U, Kremp S, Schaefer-Schuler A, et al. Comparison of different methods for delineation of 18F-FDG PET-positive tissue for target volume definition in 
radiotherapy of patients with non-Small cell lung cancer. J Nucl Med 2005;46:1342-1348.

19. Hong R, Halama J, Bova D, et al. Correlation of PET standard uptake value and CT window-level thresholds for target delineation in CT-based radiation treatment planning. Int J Radiat Oncol Biol Phys 2007;67:720-726.

20. Burri RJ, Rangaswamy B, Kostakoglu L, et al. Correlation of positron emission tomography standard uptake value and pathologic specimen size in cancer of the head and neck. Int J Radiat Oncol Biol Phys 2008;71:682-688.

21. Koshy M, Paulino AC, Howell R, et al. F-18 FDG PET-CT fusion in radiotherapy treatment planning for head and neck cancer. Head Neck 2005;27:494-502.

22. Bradley J, Thorstad WL, Mutic S, et al. Impact of FDG-PET on radiation therapy volume delineation in non-small-cell lung cancer. Int J Radiat Oncol Biol Phys 2004;59:78-86.

23. Rembielak A, Price P. The role of PET in target localization for radiotherapy treatment planning. Onkologie 2008;31:57-62.

24. Gregoire V, Bol A, Geets X, et al. Is PET-based treatment planning the new standard in modern radiotherapy? The head and neck paradigm. Semin Radiat Oncol 2006;16:232-238. 
25. Gregoire V, Haustermans K, Geets X, et al. PET-based treatment planning in radiotherapy: a new standard? J Nucl Med 2007;48 Suppl 1:68S-77S.

26. Troost EG, Schinagl DA, Bussink J, et al. Innovations in radiotherapy planning of head and neck cancers: role of PET. J Nucl Med;51:66-76. 


\section{FIGURE LEGENDS}

\section{Figure 1}

(a) Brain semiconductor PET image and (b) whole-body BGO scintillator PET image from patient No. 5 with a T3N2M0 nasopharyngeal carcinoma. On the brain semiconductor PET image, the boundary of tumor uptake is more clearly identified. (c) Radiotherapy treatment plan based on GTV NEW $_{(P L A N}$ NEW $_{\text {) and (d) Radiotherapy }}$ treatment plan based on $\mathrm{GTV}_{\mathrm{CONV}}\left(\mathrm{PLAN}_{\mathrm{CONV}}\right)$ from the same patient. Blue, aqua, and orange lines show $2000 \mathrm{cGy}, 1600 \mathrm{cGy}$, and $1000 \mathrm{cGy}$ isodose lines, respectively. The red line indicates $\mathrm{PTV}_{\mathrm{NEW}}$, while the green line indicates $\mathrm{PTV}_{\mathrm{CONV}}$.

\section{Figure 2}

Dose-volume histograms of PLAN $\mathrm{NEW}_{\text {(solid line) and PLAN }}$ CONV $_{\text {(dashed line) shown }}$ in Fig.1 for (a) brain stem and (b) cerebrum and cerebellum. 
Table 1. Patient characteristics

\begin{tabular}{|c|c|c|c|c|}
\hline Patient No. & Sex & Age & T stage & N stage \\
\hline 1 & M & 30 & $\mathrm{~T} 3$ & $\mathrm{~N} 2$ \\
\hline 2 & M & 61 & $\mathrm{~T} 3$ & $\mathrm{~N} 3 \mathrm{~b}$ \\
\hline 3 & $\mathrm{~F}$ & 35 & $\mathrm{~T} 4$ & N1 \\
\hline 4 & M & 53 & $\mathrm{~T} 2 \mathrm{~b}$ & N1 \\
\hline 5 & $\mathrm{~F}$ & 55 & $\mathrm{~T} 3$ & $\mathrm{~N} 2$ \\
\hline 6 & $\mathrm{M}$ & 61 & $\mathrm{~T} 2 \mathrm{a}$ & $\mathrm{N} 2$ \\
\hline 7 & $\mathrm{~F}$ & 67 & $\mathrm{~T} 2 \mathrm{a}$ & $\mathrm{N} 1$ \\
\hline 8 & M & 76 & $\mathrm{~T} 2 \mathrm{~b}$ & $\mathrm{~N} 2$ \\
\hline 9 & $\mathrm{M}$ & 60 & $\mathrm{~T} 1$ & $\mathrm{~N} 2$ \\
\hline 10 & $\mathrm{M}$ & 53 & $\mathrm{~T} 1$ & N1 \\
\hline 11 & $\mathrm{~F}$ & 71 & $\mathrm{~T} 3$ & No \\
\hline 12 & $\mathrm{M}$ & 61 & $\mathrm{~T} 2 \mathrm{~b}$ & $\mathrm{~N} 2$ \\
\hline
\end{tabular}


Table 2. Absolute volume of GTV

\begin{tabular}{|c|c|c|c|}
\hline Patient No. & $\operatorname{GTV}_{\text {NEW }}(\mathrm{ml})$ & $\operatorname{GTV}_{\text {CONV }}(\mathrm{ml})$ & Time course \\
\hline 1 & 27.9 & 63.0 & Protocol 1 \\
\hline 2 & 31.6 & 44.9 & Protocol 1 \\
\hline 3 & 23.4 & 26.4 & Protocol 1 \\
\hline 4 & 9.8 & 20.6 & Protocol 1 \\
\hline 5 & 27.8 & 75.9 & Protocol 1 \\
\hline 6 & 20.8 & 52.6 & Protocol 1 \\
\hline 7 & 8.9 & 22.3 & Protocol 1 \\
\hline 8 & 6.7 & 17.8 & Protocol 2 \\
\hline 9 & 4.9 & 16.5 & Protocol 2 \\
\hline 10 & 5.3 & 10.6 & Protocol 2 \\
\hline 11 & 9.1 & 25.2 & Protocol 2 \\
\hline 12 & 12.6 & 31.7 & Protocol 2 \\
\hline Average \pm SD & $15.7 \pm 9.9$ & $34.0 \pm 20.5$ & \\
\hline P values & \multicolumn{2}{|c|}{0.0006} & \\
\hline
\end{tabular}

Abbreviations: $\mathrm{SD}=$ standard deviation 
Table 3. The maximum and mean dose of PTV

\begin{tabular}{|c|c|c|c|c|}
\hline \multirow{2}{*}{ Patient No. } & \multicolumn{2}{|c|}{ Maximum dose of PTV (cGy) } & \multicolumn{2}{|c|}{ Mean dose of PTV (cGy) } \\
\hline & PLAN $_{\text {NEW }}$ & PLAN $_{\text {CONV }}$ & PLAN $_{\text {NEW }}$ & PLAN $_{\text {CONV }}$ \\
\hline 1 & 2376 & 2422 & 2150 & 2179 \\
\hline 2 & 2285 & 2329 & 2139 & 2157 \\
\hline 3 & 2261 & 2310 & 2121 & 2148 \\
\hline 4 & 2398 & 2462 & 2182 & 2190 \\
\hline 5 & 2275 & 2254 & 2130 & 2116 \\
\hline 6 & 2286 & 2312 & 2125 & 2140 \\
\hline 7 & 2432 & 2442 & 2218 & 2215 \\
\hline 8 & 2265 & 2227 & 2133 & 2118 \\
\hline 9 & 2208 & 2216 & 2112 & 2118 \\
\hline 10 & 2337 & 2335 & 2165 & 2158 \\
\hline 11 & 2329 & 2326 & 2184 & 2171 \\
\hline 12 & 2248 & 2301 & 2136 & 2147 \\
\hline Average \pm SD & $2308 \pm 67$ & $2328 \pm 79$ & $2150 \pm 32$ & $2155 \pm 31$ \\
\hline Pvalues & & & & \\
\hline
\end{tabular}

Abbreviations: $\mathrm{PTV}=$ Planning Target Volume, $\mathrm{SD}=$ standard deviation 
Table 4. The maximum dose of cerebrum and cerebellum and brain stem

\begin{tabular}{|c|c|c|c|c|}
\hline \multirow{2}{*}{ Patient No. } & \multicolumn{2}{|c|}{ Cerebrum and Cerebellum (cGy) } & \multicolumn{2}{|c|}{ Brain Stem (cGy) } \\
\hline & PLAN $_{\text {NEW }}$ & PLAN $_{\text {CONV }}$ & PLAN $_{\text {NEW }}$ & PLAN $_{\text {CONV }}$ \\
\hline 1 & 2182 & 2340 & 1895 & 2176 \\
\hline 2 & 2224 & 2333 & 2137 & 2191 \\
\hline 3 & 2260 & 2310 & 2186 & 2189 \\
\hline 4 & 1278 & 2377 & 1223 & 1879 \\
\hline 5 & 2227 & 2246 & 2072 & 2197 \\
\hline 6 & 1737 & 1627 & 1327 & 2011 \\
\hline 7 & 2430 & 2442 & 1371 & 1603 \\
\hline 8 & 1878 & 2196 & 1068 & 1613 \\
\hline 9 & 1860 & 2164 & 980 & 1532 \\
\hline 10 & 2056 & 2163 & 586 & 664 \\
\hline 11 & 2329 & 2326 & 2243 & 2173 \\
\hline 12 & 1555 & 2274 & 606 & 1564 \\
\hline Average \pm SD & $2001 \pm 347$ & $2233 \pm 209$ & $1475 \pm 612$ & $1816 \pm 455$ \\
\hline$P$ values & & & & \\
\hline
\end{tabular}

Abbreviations: $\mathrm{SD}=$ standard deviation 


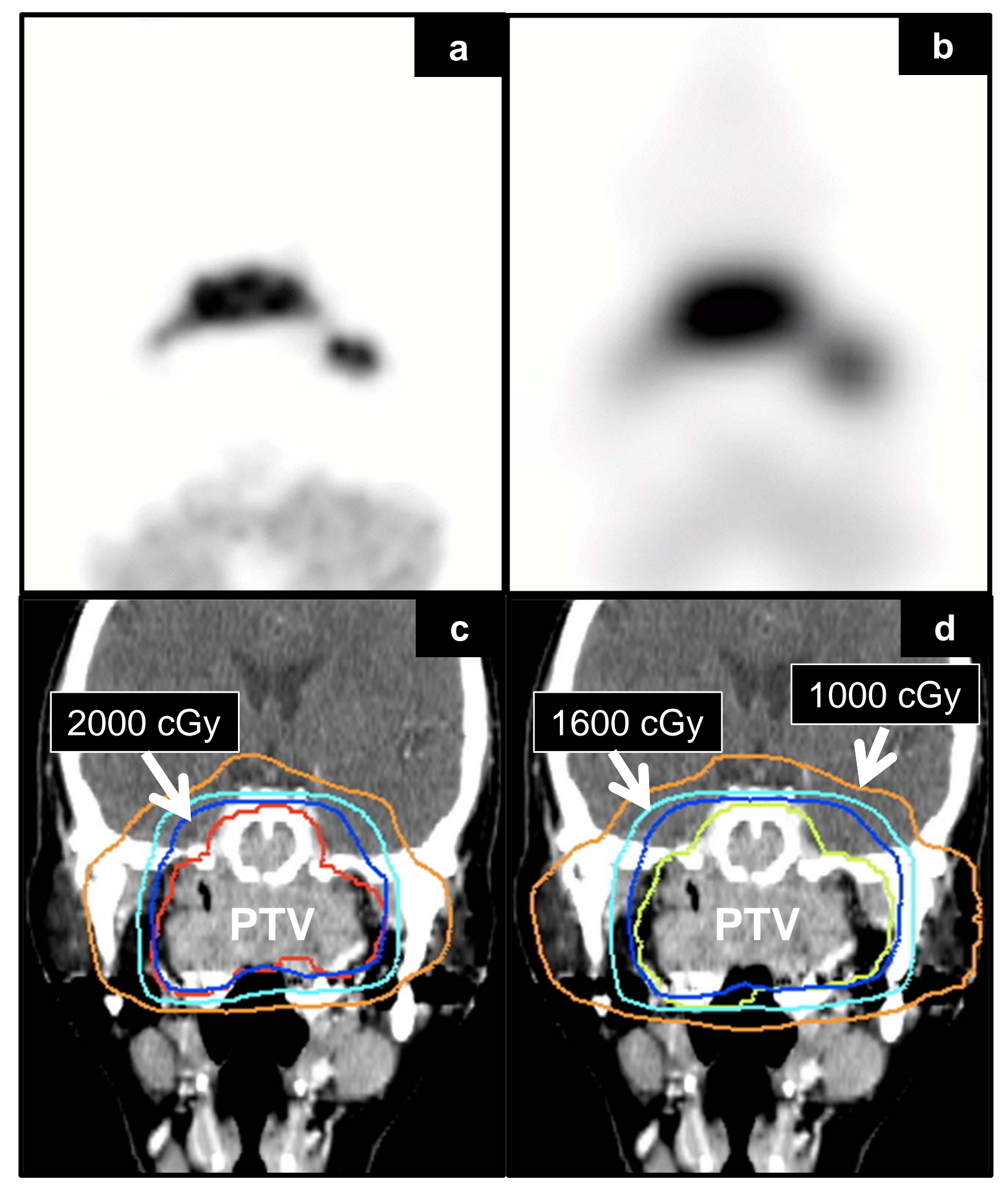



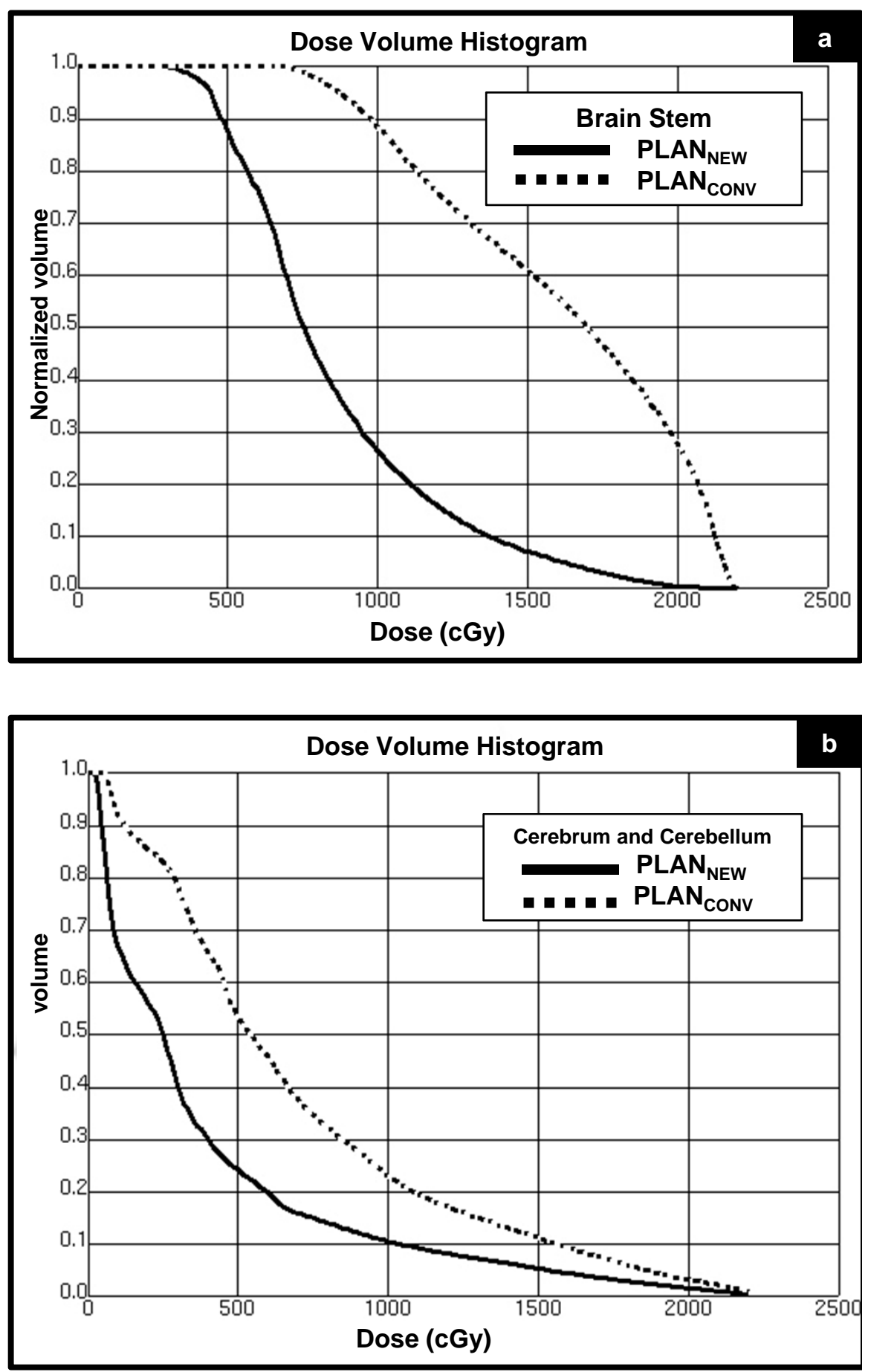